\title{
Neonatal immune activation during early and late postnatal brain development differently influences depression-related behaviors in adolescent and adult C57BL/6 mice
}

\author{
Jafar Majidi-Zolbanin ${ }^{1}$, Mohammad-Hossein Doosti ${ }^{1}$, Behzad Baradaran ${ }^{1}$, Mohammad Amani², \\ Maryam Azarfarin ${ }^{3}$, Ali-Akbar Salari ${ }^{3,4}$ \\ ${ }^{1}$ Immunology Research Center, Tabriz University of Medical Sciences, Tabriz 51639, East Azerbaijan, Iran. \\ ${ }^{2}$ Department of Physiology, Faculty of Medicine, Ardabil University of Medical Sciences, Ardabil 55136, Ardabil, Iran. \\ ${ }^{3}$ Drug Applied Research Center, Tabriz University of Medical Sciences, Tabriz 51639, East Azerbaijan, Iran. \\ ${ }^{4}$ Laboratory of Neuropsychopharmacology and Psychoneuroimmunology, Hayyan Research Institute, University of Tabriz, Tabriz \\ 51639, East Azerbaijan, Iran.
}

\section{A B S T R A C T}

Aim: Immune challenge during early and late neonatal periods can induce robust alterations in physiological and behavioral functions, resulting in greater risk for the development of neuropsychiatric disorders, such as anxiety and depression, later in life. In addition, previous studies concluded that increasing age correlates with increased depression behaviors in humans and rodents. This study aimed to investigate for the first time whether immune challenge with a viral mimic, synthetic double-stranded ribonucleic acid (Poly I: C) during different neonatal periods can differently affect depression-related behaviors in adolescent and adult mice. Methods: Male C57BL/6 mice were treated with either saline or Poly I:C (1 mg/kg and $4 \mathrm{mg} / \mathrm{kg}$ ) on postnatal days (PND) 3-5 (early neonatal phase) or PND 14-16 (late neonatal phase), and then subjected to behavioral tests, including tail suspension test and forced swimming test, during adolescence (PND 35 or 40) and adulthood (PND 85 or 90). Results: The results demonstrated that early neonatal immune activation increases depression-related behaviors in both adolescent and adult mice, but late neonatal immune activation only increases depression in adult mice. In other words, these findings indicated that the nature of the offspring's neuropathology can depend on the severity of the insult, the pup's age at the time of the insult, and offspring age at the time of behavioral testing. Conclusion: These findings suggest that dose and timing of neonatal insult and offspring age may be important factors for evaluating neuropsychiatric disorders in adults who experienced early life infection.

Key words: Age, depression, hypothalamic-pituitary-adrenal axis, mice, neonatal infection, Poly I:C

\section{INTRODUCTION}

Many studies have recently demonstrated the importance of early life infection on the brain and behavior development, and how such infections can

\begin{tabular}{|l|l|}
\hline \multicolumn{2}{|c|}{ Access this article online } \\
\hline Quick Response Code: & \\
\hline & Website: \\
\hline & www.nnjournal.net \\
\cline { 2 - 2 } & \\
\hline
\end{tabular}

increase susceptibility to the onset of neuropsychiatric disorders, such as anxiety and depression, later in life. ${ }^{[1-7]}$ Lipopolysaccharide (LPS, mimics bacterial infection) and polyinosinic-polycytidylic acid (Poly I:C, mimics viral infection) administration during the neonatal period are known as two animal models of neonatal infection, and both can stimulate the immune and endocrine systems. ${ }^{[8]}$ We and others have shown that LPS- or Poly I:C-induced neonatal infection can lead to hypothalamic-pituitary-adrenal (HPA) axis abnormalities and results in increased anxiety- and depression-like behaviors in adult rodents. ${ }^{[6,7,9]}$

Corresponding Author: Mr. Ali-Akbar Salari, Laboratory of Neuropsychopharmacology and Psychoneuroimmunology, Hayyan Research Institute, University of Tabriz, Tabriz 51639, East Azerbaijan, Iran. E-mail: aa.salari@yahoo.com 
Scientific reports have indicated that the nature of the offspring's neuropathology can depend on the nature and severity of insult and the pup's age at the time of insult. This argument is supported by previous studies in which we and others showed that the time points of prenatal and neonatal immune activation, the offspring's age, and the dose of immunogen can be important factors for assessing neuropsychological alterations such as anxiety- and depression-related behaviors that persist until adulthood in mice and rats. ${ }^{[3,6,10,11]}$ For instance, a study conducted by Walker et al. ${ }^{[11]}$ indicated that postnatal immune challenge had no impact on anxiety levels in adolescence, but did lead to increased levels of anxiety in adulthood and senescence in male rats. Furthermore, previous studies concluded that older age correlates with an increased level of depression behavior in humans and rodents. ${ }^{[10,12]}$ However, limited information is available regarding the relationship between the timing of neonatal immune challenge and age in development of depression-related behaviors in adulthood.

Therefore, the aim of the current study was to investigate the impacts of neonatal immune activation with equal Poly I:C doses at two time points, postnatal days (PND) 3-5 (early neonatal phase) or PND 14-16 (late neonatal phase), on depression-related behaviors in adolescent and adult male mice.

\section{METHODS}

\section{Animals}

Male and female C57BL/6 mice (70-80 days) were obtained from the animal house of Pasteur Institute (Tehran, Iran). Mice were housed in standard polycarbonate cages in a room with a $12: 12 \mathrm{~h}$ light/dark cycle (lights on 08:00-20:00), controlled temperature $\left(23 \pm 1^{\circ} \mathrm{C}\right)$, and had free access to food and water. These conditions were kept as a standard housing condition in all stages of experiments. Following a 2-week period of acclimatization to the new animal housing room, male and female mice were kept together one-by-one in a cage to facilitate mating. Female mice were visually monitored daily for confirmation of pregnancy. Once confirmed, the female mice were removed from the breeding cages and housed individually in standard cages. ${ }^{[10,13]}$ All pregnant mice were allowed to have a normal delivery, and the first day of birth was considered PND $0 .{ }^{[6]}$ One day after the birth, all litters were culled to four pups per mother. On day 21, litters were weaned by removal of the mother, and only male pups were used in this study. A total of
30 litters were used during this study in three stages, each of which included 10 litters. Only one mouse per dam was selected for each of the groups to avoid the litter effect. All procedures of the study were performed in accordance with the ethical guidelines set by the Research and Ethics Committee of the Tabriz University of Medical Sciences, which completely adhere with the National Institutes of Health NIH Guide for the Care and Use of Laboratory Animals (NIH; Publication No. 85-23, revised 1985).

\section{Neonatal immune activation}

A summary of the experimental design is shown in Figure 1. The pups were divided based on treatment conditions into two clusters: group 1 - saline-injected mice and group 2 - Poly I:C-injected mice (each group only used for two tests with a 5-day interval between each test; $n=8$ per group). The dams were removed from their pups for approximately $5 \mathrm{~min}$, and the pups were weighed and received a subcutaneous (in the interscapular region) injection of Poly I:C (Sigma Aldrich, St Louis, MO, USA; $1 \mathrm{mg} / \mathrm{kg}$ and $4 \mathrm{mg} / \mathrm{kg}$ ) or saline $(1 \mathrm{~mL} / \mathrm{kg})$ during the early neonatal phase (PND 3-5, which corresponds to the third trimester of human pregnancy when major brain growth occurs) or the late neonatal phase (PND 14-16, which corresponds to 1-2 years old humans). ${ }^{[6,14]}$ The doses and timing of Poly I:C treatment were chosen based on previous studies. ${ }^{[6,8,15]}$ The Poly $\mathrm{I}: \mathrm{C}$ was dissolved in sterile saline $(0.9 \% \mathrm{NaCl})$, and injections were performed between 10:00 and 11:00. Each injection was performed through a 27 -gauge needle connected by polyethylene tubing to a $10-\mu \mathrm{L}$ Hamilton syringe. Newborn mice were returned to their housing immediately after injections.

\section{Behavioral tests}

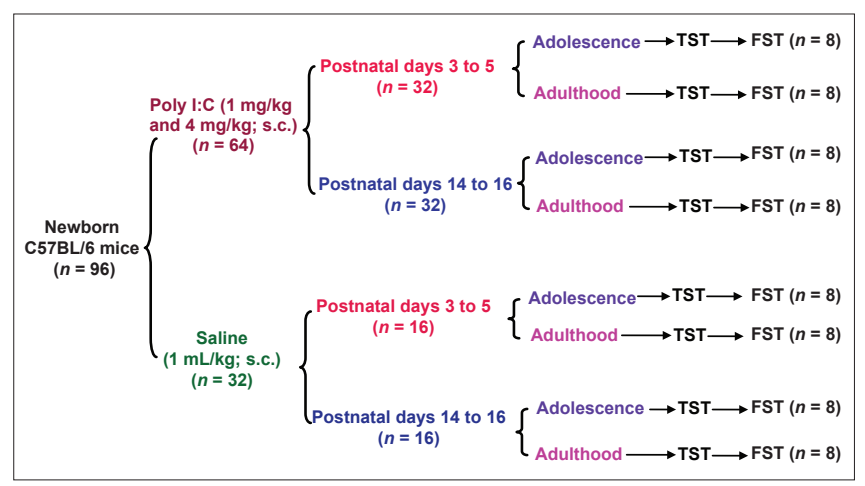

Figure 1: Experimental design: The effects of early and late neonatal immune activation with Poly I:C on depression-related behaviors during adolescence and adulthood in the tail suspension test (TST) and forced swimming test (FST) in C57BL/6 mice 
All behavioral parameters were recorded by observers who were blind to the treatment. In addition, all behavioral tests were conducted in a quiet room during the light period (between 13:00 and 18:00) under bright and moderate illumination, and the mice were kept in the room for at least $1 \mathrm{~h}$ before the assessment. Depression-related behavior was separately studied in adolescent (PND 35: tail suspension test [TST]; PND 40: forced swimming test [FST]) and adult (PND 85: TST; PND 90: FST) male mice.

TST: The TST was performed according to the previously described procedure ${ }^{[6]}$ At the beginning of the experiment, each mouse was individually suspended by its tail using a clamp, $2 \mathrm{~cm}$ from the distal end, for $5 \mathrm{~min}$ in a gray wooden box ( $40 \mathrm{~cm}$ high, $30 \mathrm{~cm}$ wide, and $20 \mathrm{~cm}$ deep), with the head about $25 \mathrm{~cm}$ above the floor. The total duration of immobility was recorded (in seconds). All animals that climbed their tails during the TST were excluded from the further analyses. Immobility was defined as the lack of whole-body motion, whereas mobility was defined as hind leg movement. ${ }^{[16]}$

FST: The FST remains one of the most widely used tools for measuring behavioral despair in rodents. To describe this behavioral model in mice, the following procedure was adopted: mice were individually placed into the transparent glass cylinders (height: $25 \mathrm{~cm}$, diameter: $10 \mathrm{~cm}$ ) filled with water to a height of $15 \mathrm{~cm}$ and maintained at $25 \pm 1^{\circ} \mathrm{C}$. The water was replaced between each test. The total duration of immobility was recorded during the last 4 min of the 6-min testing period. At the end of swimming session, the animals were removed from the cylinder, dried with towels, and gently placed near an electric heater for 15-30 min. Each mouse was judged to be immobile when it ceased struggling and remained floating motionless in the water, making only those movements necessary to keep its head above water. A decrease in the duration of immobility time is considered indicative of depression-like behavior in mice. ${ }^{[6,10]}$

\section{Statistical analysis}

The statistical analysis was performed using Statistical Package for Social Sciences software (Version 21, IBM, Armonk, NY, USA). The depression results were analyzed using three-way analysis of variance, with age, treatment, and neonatal infection timing as the main factors. All data are presented as the mean \pm standard error of the mean. Further analysis was carried out using Tukey's honest significant different post-hoc tests for multiple comparisons. $P<0.05$ was considered as statistically significant.

\section{RESULTS}

Effects of early and late neonatal immune activation on depression-related behaviors during adolescence and adulthood in the TST

The three-way analysis revealed the significant effects of the time of neonatal immune activation $\left(F_{1,84}=5.65\right.$, $P<0.03)$, age $\left(F_{1.84}=43.03, P<0.001\right)$, and treatment $\left(F_{2,84}=11.57, P<0.001\right)$ on the immobility time in the TST. Significant interactions existed between age $\times$ treatment $\left(F_{2,84}=4.66, P<0.02\right)$ and the time of neonatal immune activation $\times$ age $\times$ treatment $\left(F_{2,84}=3.37, P<0.04\right)$. However, there was no significant interaction between the time of neonatal immune activation $\times$ age and the time of neonatal immune activation $\times$ treatment. These results indicate that neonatal immune activation with Poly I:C can influence depression-related behaviors in dose-, age-, and time-dependent manner in mice. Therefore, the dose of immunogen, the timing of immune activation, and age may be important factors for evaluating the consequences of neonatal immune activation on affective disorders, like depression, later in life.

The data analysis indicated that early neonatal immune activation with Poly I: C increased the total duration of immobility at the dose of $4 \mathrm{mg} / \mathrm{kg}$ in adolescence [Figure 2a; $P=0.037$ ] and at both doses in adulthood [Figure 2b; $P=0.042$ and $P=0.002$ ], indicating high levels of depression-related behaviors in Poly I:C-treated mice in comparison with the saline-treated group.

As shown in Figure 3, late neonatal immune challenge with Poly I:C resulted in an increase in the total duration of immobility time at the dose of $4 \mathrm{mg} / \mathrm{kg}$ in adulthood $[P=0.03]$, but not in adolescence [Figure 3].

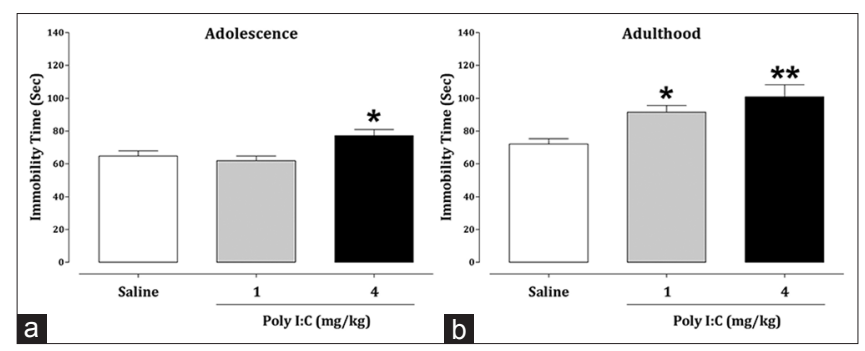

Figure 2: Effects of early neonatal immune activation on depression-like behavior during adolescence (a) and adulthood (b) in the tail suspension test. The data are presented as mean \pm standard error of the mean $(n=8)$. ${ }^{*} P<0.05$ and ${ }^{\star *} P<0.01$ compared with the saline-treated group 
In addition, we found high levels of depression-related behaviors in adulthood in Poly I:C-exposed mice compared with saline-injected mice.

Effects of early and late neonatal immune activation on depression-related behaviors during adolescence and adulthood in the FST

The three-way analysis indicated the significant effects of the time of postnatal immune activation $\left(F_{1,84}=34.69\right.$, $P<0.001)$, age $\left(F_{1,4}=20.37, P<0.001\right)$, and treatment $\left(F_{2,84}=20.53, P<0.001\right)$ on the immobility time in the FST. Considerable interactions existed between the time of neonatal immune activation $\times$ treatment $\left(F_{2,84}=5.02, P<0.009\right)$ and age $\times$ treatment $\left(F_{2,84}=5.77, P<0.005\right)$. However, there was no interaction between the time of neonatal immune activation $\times$ age and the time of neonatal immune activation $\times$ age $\times$ treatment. These data demonstrate that immune activation with Poly I:C during postnatal brain development can affect depression-related behaviors in dose-, age-, and time-dependent manner in adult mice. Thus, these different factors may affect the effects of neonatal immune activation on affective disorders later in life in animal models.

The results of the FST assessment showed that early postnatal immune activation with Poly I:C increased immobility time at the dose of $4 \mathrm{mg} / \mathrm{kg}$ during adolescence [Figure 4a; $P=0.015$ ] and at both doses in adulthood [Figure $4 \mathrm{~b} ; P=0.002$ and $P=0.000$ ]. Higher levels of depression-related behaviors were measured in Poly I:C-treated mice in comparison with the saline-treated group.

Our data also showed that immune activation with $4 \mathrm{mg} / \mathrm{kg}$ Poly I:C during late neonatal brain development increased the total duration of immobility in adulthood ( $P=0.019)$, but not in adolescence [Figure 5], indicating high levels of depression-related behaviors during adulthood in Poly I:C-exposed mice relative to the saline-treated group. These results confirmed that time of postnatal immune challenge, age and the dose of immunogen can be important factors for evaluating depression-related behaviors in mice.

\section{DISCUSSION}

We recently showed that early postnatal immune challenge with the bacterial endotoxin and LPS can lead to increased levels of corticosterone (COR) and depression-like symptoms in adult male and female NMRI mice. ${ }^{[6]}$ In addition, early postnatal immune challenge has been shown to have adverse outcomes on

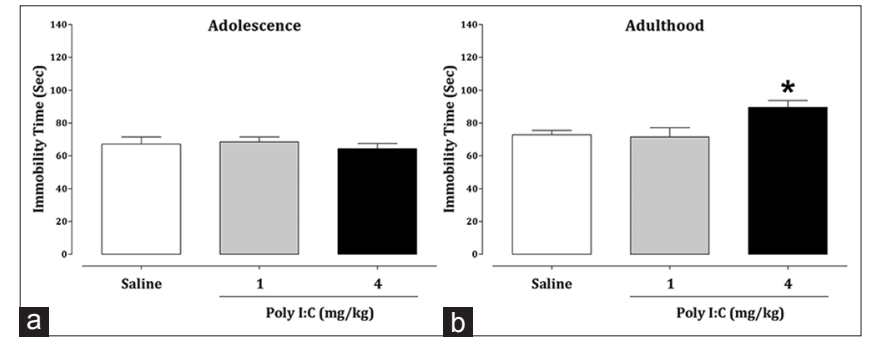

Figure 3: Effects of late neonatal immune challenge on depression-like behavior during adolescence (a) and adulthood (b) in the tail suspension test. The data are presented as mean \pm standard error of the mean $(n=8)$. ${ }^{*} P<0.05$ compared to the saline-treated group

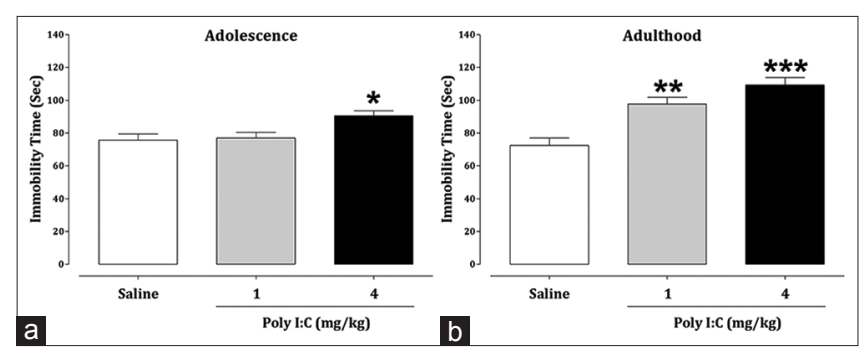

Figure 4: Impacts of early postnatal immune activation on depression-like behavior during adolescence (a) and adulthood (b) in the forced swimming test. The data are presented as mean \pm standard error of the mean $(n=8) .{ }^{*} P<0.05$, ${ }^{\star *} P<0.01$, and ${ }^{* * *} P<0.001$ compared to the saline-treated group

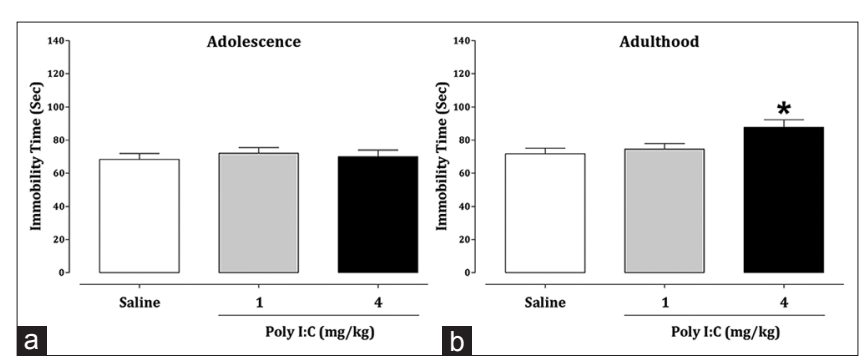

Figure 5: Effects of late neonatal immune activation on depression-like behavior during adolescence (a) and adulthood (b) in the forced swimming test. The data are presented as mean \pm standard error of the mean $(n=8) .{ }^{*} P<0.05$, compared to the saline-treated group

physiological, behavioral, and neuroendocrine systems in adulthood. ${ }^{[6,8,9]}$ For instance, it has been reported that bacterial and viral infections during early ${ }^{[9,11]}$ and late ${ }^{[15]}$ neonatal periods results in increased anxiety-like behaviors and disrupted HPA axis activity in adult rodents. Our results demonstrated that early neonatal immune activation led to increased depression-related behaviors in both adolescent and adult mice, but late neonatal infection only increased depression in adult mice. In this regard, Konat et al. ${ }^{[15]}$ showed that anxiety levels in rats following late postnatal immune activation were much larger than those observed by Ibi et al. ${ }^{[17]}$ in mice following early neonatal immune challenge using a similar behavioral testing. Previous studies demonstrated that early postnatal immune challenge increased baseline COR levels during adolescence and adulthood, while late neonatal immune activation did not alter baseline COR levels in adulthood. ${ }^{[18]}$ It was 
also found that COR suppressed cell proliferation and neurogenesis in the hippocampus, which can induce depression-like behaviors. ${ }^{[19]}$ Moreover, the important role of the hippocampus in depression-related behaviors has been shown in humans and rodents. ${ }^{[13]}$ In line with this, we demonstrated that adolescent fluoxetine treatment reduced depression-like behaviors induced by early neonatal infection in adult mice ${ }^{[6]}$ It has been reported that chronic fluoxetine and imipramine treatments prevent the COR-induced reduction in cell proliferation and activates neurogenesis in the hippocampus. ${ }^{[19]}$ It seems reasonable to speculate that an increase in depression-related behaviors or baseline COR during adolescence following early neonatal immune activation may further suppress cell proliferation and neurogenesis in the hippocampus in comparison with late neonatal immune activation, and these effects can increase the severity of depression in adulthood. Moreover, we observed an interaction between treatment, age, and the time of neonatal immune activation on depression-related behaviors in mice. Poly I:C at the dose of $4 \mathrm{mg} / \mathrm{kg}$ during early postnatal brain development increased depression-related behaviors in adolescent mice, while the same dose during late neonatal phase had no significant effect on depression in adolescent mice. Notably, the mice treated with Poly I:C at both doses during early neonatal period exhibited elevated depression-related behaviors as adults, while only the dose of $4 \mathrm{mg} / \mathrm{kg}$ during the late neonatal phase increased depression in adult mice. Taken together, the findings of this study suggest that the effect of neonatal immune activation on depression-related behaviors in mice is dependent on the timing of the immune challenge and the dose of immunogen.

\section{REFERENCES}

1. Wu XQ, Li XF, Ye B, Popat N, Milligan SR, Lightman SL, O'Byrne KT. Neonatal programming by immunological challenge: effects on ovarian function in the adult rat. Reproduction 2011;141:241-8.

2. Korosi A, Naninck EF, Oomen CA, Schouten M, Krugers H, Fitzsimons C, Lucassen PJ. Early-life stress mediated modulation of adult neurogenesis and behavior. Behav Brain Res 2012;227:400-9.

3. Spencer SJ, Galic MA, Pittman QJ. Neonatal programming of innate immune function. Am J Physiol Endocrinol Metab 2011;300:E11-8.

4. Asiaei M, Solati J, Salari AA. Prenatal exposure to LPS leads to long-lasting physiological consequences in male offspring. Dev Psychobiol 2011;53:828-38.

5. Babri S, Doosti MH, Salari AA. Strain-dependent effects of prenatal maternal immune activation on anxiety- and depression-like behaviors in offspring. Brain Behav Immun 2014;37:164-76.

6. Doosti MH, Bakhtiari A, Zare P, Amani M, Majidi-Zolbanin N, Babri S, Salari AA. Impacts of early intervention with fluoxetine following early neonatal immune activation on depression-like behaviors and body weight in mice. Prog Neuropsychopharmacol Biol Psychiatry 2013;43:55-65

7. Majidi-Zolbanin J, Azarfarin M, Samadi H, Enayati M, Salari AA. Adolescent fluoxetine treatment decreases the effects of neonatal immune activation on anxiety-like behavior in mice. Behav Brain Res 2013;250:123-32.

8. Ellis S, Mouihate A, Pittman QJ. Neonatal programming of the rat neuroimmune response: stimulus specific changes elicited by bacterial and viral mimetics. $J$ Physiol 2006;571:695-701.

9. Sominsky L, Fuller EA, Bondarenko E, Ong LK, Averell L, Nalivaiko E, Dunkley PR, Dickson PW, Hodgson DM. Functional programming of the autonomic nervous system by early life immune exposure: implications for anxiety. PLoS One 2013;8:e57700.

10. Enayati M, Solati J, Hosseini MH, Shahi HR, Saki G, Salari AA. Maternal infection during late pregnancy increases anxiety- and depression-like behaviors with increasing age in male offspring. Brain Res Bull 2012;87:295-302.

11. Walker FR, March J, Hodgson DM. Endotoxin exposure in early life alters the development of anxiety-like behaviour in the Fischer 344 rat. Behav Brain Res 2004;154:63-9.

12. Osborn DP, Fletcher AE, Smeeth L, Stirling S, Bulpitt CJ, Breeze E, $\mathrm{Ng}$ ES, Nunes M, Jones D, Tulloch A. Factors associated with depression in a representative sample of 14217 people aged 75 and over in the United Kingdom: results from the MRC trial of assessment and management of older people in the community. Int $J$ Geriatr Psychiatry 2003;18:623-30.

13. Krishnan V, Nestler EJ. The molecular neurobiology of depression. Nature 2008;455:894-902.

14. Avishai-Eliner S, Brunson KL, Sandman CA, Baram TZ. Stressed-out, or in (utero)? Trends Neurosci 2002;25:518-24.

15. Konat GW, Lally BE, Toth AA, Salm AK. Peripheral immune challenge with viral mimic during early postnatal period robustly enhances anxiety-like behavior in young adult rats. Metab Brain Dis 2011;26:237-40.

16. Amani M, Samadi H, Doosti MH, Azarfarin M, Bakhtiari A, Majidi-Zolbanin N, Mirza-Rahimi M, Salari AA. Neonatal NMDA receptor blockade alters anxiety- and depression-related behaviors in a sex-dependent manner in mice. Neuropharmacology 2013;73:87-97

17. Ibi D, Nagai T, Kitahara Y, Mizoguchi H, Koike H, Shiraki A, Takuma K, Kamei H, Noda Y, Nitta A, Nabeshima T, Yoneda Y, Yamada K. Neonatal PolyI:C treatment in mice results in schizophrenia-like behavioral and neurochemical abnormalities in adulthood. Neurosci Res 2009;64:297-305.

18. Soriano RN, Branco LG. Reduced stress fever is accompanied by increased glucocorticoids and reduced PGE2 in adult rats exposed to endotoxin as neonates. $J$ Neuroimmunol 2010;225:77-81.

19. Murray F, Smith DW, Hutson PH. Chronic low dose corticosterone exposure decreased hippocampal cell proliferation, volume and induced anxiety and depression like behaviours in mice. Eur $J$ Pharmacol 2008;583:115-27.

Cite this article as: Majidi-Zolbanin J, Doosti MH, Baradaran B, Amani M Azarfarin M, Salari AA. Neonatal immune activation during early and late postnatal brain development differently influences depression-related behaviors in adolescent and adult C57BL/6 mice. Neuroimmunol Neuroinflammation 2014;1(1):35-9.

Source of Support: Nil. Conflict of Interest: No.

Received: 28-04-2014; Accepted: 30-05-2014 